\title{
High Energy Particle Accelerators*
}

\author{
K. M. Terwilliger \\ The University of Michigan, Ann Arbor, Michigan
}

\section{INTRODUCTION}

In this paper I will describe some of the principles of the multi-Bev particle accelerators in existence today and being seriously considered for the future. As examples, I shall discuss mainly the Bevatron at the Lawrence Radiation Laboratory at Berkeley, which is a $6 \mathrm{Bev}$ proton synchrotron of the weak focusing type, the AGS (Alternating Gradient Synchrotron) at Brookhaven National Laboratory, a 30 Bev strong focusing proton synchrotron, and FFAG (Fixed Field Alternating Gradient) accelerator models being studied by MURA, the Midwestern Universities Research Association, at Madison, Wisconsin.

\section{WEAK Focusing Synchrotrons}

A schematic layout of the Bevatron is shown in Fig. 1.** Protons from an ion source are accelerated to about $0.5 \mathrm{Mev}$ by a CockroftWalton, then to $10 \mathrm{Mev}$ by a linear accelerator and injected into the Bevatron. There they are bent around in an almost circular orbit by the vertical magnetic field. They gain energy each time they pass through a gap, across which is applied an alternating voltage in phase with the particles, somewhat like the method proposed by Lawrence for the cyclotron in 1930.

There are, however, a number of differences between a cyclotron and a standard synchrotron. In a cyclotron, conducting dees shield the particles from the electric field when they are away from the accelerating gaps, while in a synchrotron the fields are confined to a small region by a radio frequency cavity. The cyclotron, also, has a magnetic field which is kept constant in time, so the particles have to spiral out to larger radii as they gain energy. In order to keep the particles at one radius, as in this synchrotron, the field must increase with time exactly as the particle momentum. There is another difference between the cyclotron and synchrotron. In the cyclotron, as long as the protons are non-relativistic, the revolution frequency, and hence the frequency of the applied accelerator voltage, is constant, independent of energy and momentum-because the particle velocity and radius both go up together. In a synchrotron, however, since the radius is kept constant, the revolution frequency, and the accelerating voltage frequency, must increase directly with the par-

\footnotetext{
* Paper presented at the CASMT Convention. November 24-26, 1960, Detroit, Michigan.
}

** Note: This presentation was accompanied by many slides. In this article, only a few have been reproduced. 
ticle velocity. When cyclotrons accelerate particles to appreciably relativistic energies, however-around $15 \mathrm{Mev}$ per nucleon-the mass changes enough to require frequency modulation here, too. These FM cyclotrons have been built to accelerate protons up to energies of $720 \mathrm{Mev}$.

The above discussion has indicated that if the applied voltage was in perfect synchronism with the particles acceleration would occur satisfactorily, as indeed it would. This would imply, however, that all the particles had been injected at one instant-and traveled around the machine in a pulse of essentially zero length-in order that all be simultaneously in phase with the applied voltage. Since the rate of injection of particles is limited, and space charge forces limit the density of particles, such a beam would be quite small. It would probably be lost, too, if perfect synchronism were required, because of the impossibility of perfect frequency tracking. Such machines, if they are to give appreciable current, must work without all particles being required to have the same phase and without perfect frequency tracking. Fortunately, the machines work without these restrictions -because they have inherent phase stability, a principle discovered independently by Veksler and McMillan in 1944 and 1945. If there is enough accelerating voltage there is a region about an equilibrium phase where the particles will undergo stable phase oscillations: a particle with the right energy but not at this equilibrium phase will gain a different voltage than a particle at this phase-giving it a different energy and hence a different frequency-sending it back toward this equilibrium phase. When it gets back there it will have a slightly wrong energy, so it will overshoot, until it is turned around and sent back again. Both the phase and energy of the particle will oscillate about their equilibrium values. So the particles do not have to start with precise energies and phases in order to be accelerated; if they start with values close to equilibrium, they will automatically remain locked-in near the equilibrium values during the acceleration. This phase-stability principle proved that both synchrotrons and synchro-cyclotrons could be practical machines-and after its discovery a number of both types were rapidly constructed.

Particles must have orbit stability, too, as well as phase stability; particles which are not headed in quite the right direction or are somewhat displaced from the design trajectory-and it is impossible to inject all the particles precisely on a given path-must be prevented from straying so far away that they hit the walls of the vacuum tank. The guiding magnetic field must be constructed so as to apply a force to these deflected particles, a force which will turn them around and head them back toward the equilibrium orbit. As in the case of the phase oscillations, the particles will then overshoot the equili- 
brium orbit, and oscillate about it- these are called betatron oscillations. This restoring force is not automatic-it must be carefully designed into the machine. If the force had the opposite sign, that is, if slightly deflected particles were always deflected further away, the beam would be completely lost. So the force must be correct with respect to sign. If the strength of the restoring force is proportional to the displacement, which it is for weak focusing machines, the betatron oscillation motion is simple harmonic--the frequency depending on the "spring constant" of the restoring force and the particle mass. If the frequency is written in terms of number of oscillations per particle revolution, only one parameter is involved, and that is the gradient of the magnetic field, defined as $n=-(r / B)(d B / d r)$. In a weak focusing machine, where the fields are the same all the way around, the number of oscillations per revolution is: radially, $Q_{r}=(1-n)^{1 / 2}$; vertically $Q_{\nabla}=n^{1 / 2}$. Clearly, for both modes of oscillation to have real frequencies, i.e., be stable, the value of $n$ is restricted to the range zero to one: that is, from the field being perfectly uniform to the field falling off in proportion to the increase in radius. Both modes then must have values of $Q$ less than 1 ; there is less than one betatron oscillation per revolution. This is why it is named weak focusing. Since the particles take more than a half revolution before they get back to the equilibrium orbit, they have a chance to travel a considerable distance away before being returned, for reasonable injection angles-requiring quite a large aperture. Since the fields change so little, the particles also have large radial excursions due to their energy variations in the phase oscillation cycle.

A cross section of the Bevatron magnet, showing the coils, vacuum tank and beam is shown in Fig. 2. The beam aperture is $1 \times 4$ feet, and it is nearly filled by the beam at injection. During the acceleration the "spring constant" of the betatron oscillation restoring force increases - and causes the amplitude of the oscillations to damp down to a considerably smaller value, as indicated in Fig. 2. The Cockroft-Walton and linear accelerator are shown in Fig. 3, and a view of the entire machine is given in Fig. 4.

The bevatron accelerates approximately $2 \times 10^{11}$ protons per pulse to $6.2 \mathrm{Bev}$, one pulse every 5 seconds. When the particles reach full energy targets are shoved into the beam. The collision of the primary energetic proton with the target nuclei produces a spray of secondary particles, some of them constituents of the nucleus, neutrons and protons, and some newly created in the collision process, mesons, hyperons, antinucleon-nucleon pairs. Fig. 5 shows a target section in the machine. Beams are made of these secondary particles, using momentum analyzing and focusing magnets. To separate particles of the same momentum but different mass, an electrostatic 


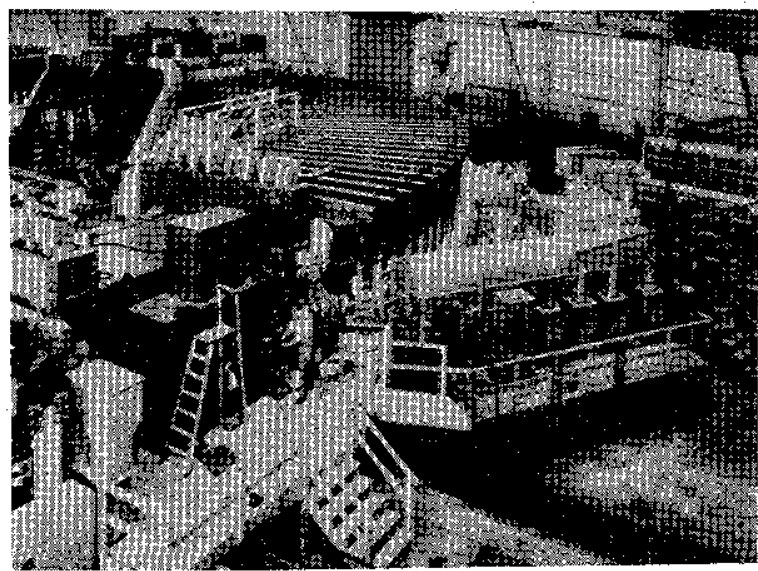

FIG. 3. Injection system of the Bevatron. CockroftWalton (right) and linear accelerator (center).

separator is used. Electric fields of strength around $100 \mathrm{kv} /$ inch can be produced over a considerable length. A separator, built at Berkeley, is shown in Fig. 6. Two such separators, combined with focusing and bending magnets are used to produce a quite pure $\mathrm{K}^{-}$ beam at the Bevatron, as seen in Fig. 7. The beam is sent into a hydrogen bubble chamber, where $\mathrm{K}^{-}$proton collisions will be photographed. Fig. 8 shows a $20^{\prime \prime}$ hydrogen bubble chamber (at Brookhaven).

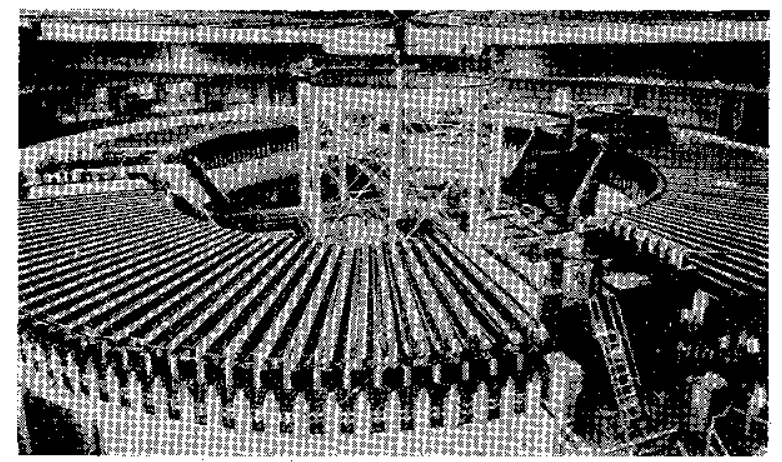

Fig. 4. Bevatron.

At the Cosmotron, a $3 \mathrm{Bev}$ proton synchrotron at Brookhaven, the primary proton beam can be deflected out of the machine into the experimental area as shown in Fig. 9. Three separate external beams are available, each of which can be separately shielded, permitting experiments to be set up while one is being run 
A number of weak focusing proton synchrotrons have been constructed or are nearing completion. The largest completed is the 10 Bev Soviet machine at Dubna (1957). It is similar to the Bevatron, only larger (and weighs 36,000 tons). Nearing completion (1962) is a $12.5 \mathrm{Bev}$ high field proton synchrotron at Argonne National Laboratory.

\section{Strong Focusing Synchrotrons}

In 1952 Courant, Livingston, and Snyder discovered the "strong focusing" principle. (It had been discovered earlier, 1950, by Christofilos, but was unpublished.) The idea is that if the parameter $n$ is made large and positive in one magnet section and large and negative in the next magnet section (rather than being constant and between zero and one, as in the weak focusing case) both the radial and vertical modes of oscillation can be made stable-with considerably more than one betatron oscillation per revolution. An optical analogy is that a focusing lens and a defocusing lens of equal strength have a net focusing action if they are spatially separated. The net focusing is less than if just the positive lens was used, of course, but it can be made as large as desired by using strong plus and minus lenses. With the increased number of betatron oscillations per revolution, the aperture required to contain these oscillations is sharply reduced. The rapidly varying field also decreases the magnitude of the radial swings produced by the phase oscillations, so the net aperture can be made quite small. Thus the iron and copper needed is only a fraction of that necessary for a similar energy weak focusing machine, and very high energy accelerators become feasible.

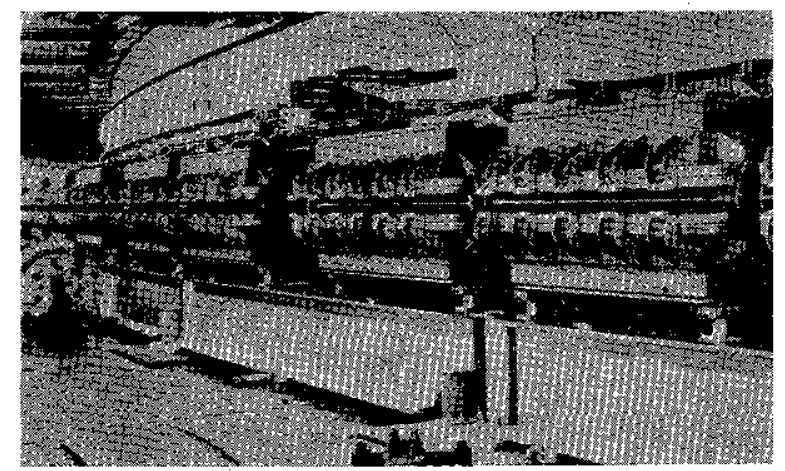

Frg. 11. Section of the AGS, with vacuum tank in place.

One of the magnet sections for the 30 Bev AGS is shown in Fig. 10. The profile of the pole tips indicates the rapid radial change of magnetic field. The aperture for the beam is only $2.7 \times 6$ inches. The 


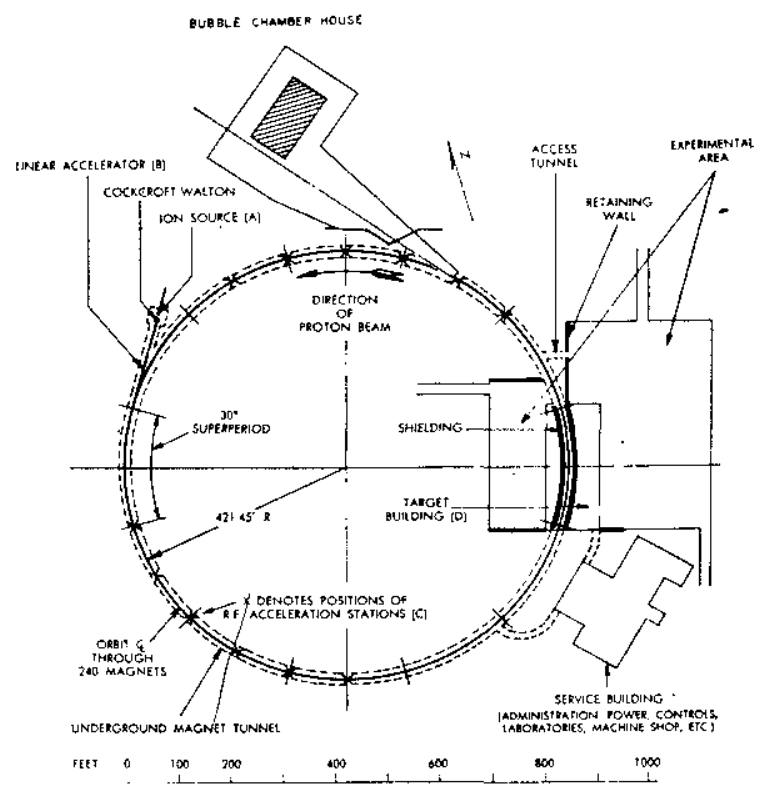

Fig. 12. Schematic plan view of the AGS.

magnitude of $n$ is 357 . Fig. 11 shows a section of the machine with the vacuum tank installed. Fig. 12 gives a schematic plan view of the accelerator. Injection is via a .75 Mev Cockroft-Walton and a $50 \mathrm{Mev}$ linear accelerator. There are $8 \frac{3}{4}$ betatron oscillations per revolution. Fig. 13 shows an aerial view of the accelerator. The machine has been run at $30 \mathrm{Bev}$ this year, and should start experiments early in 1961. The 28 Bev Proton Synchrotron at CERN, Geneva, Switzerland, the joint European accelerator, was turned on in late 1959 and is in full operation now with over $10^{11}$ protons per pulse.

\section{Other New Accelerator. Ideas}

The fixed field alternating gradient (FFAG) idea was proposed in 1953-1954 independently by Symon, Ohkawa, and Snyder, and has been developed since then mainly by the MURA group at Madison. The idea is to make a machine with a small radial aperture with the magnetic fields constant in time, rather than pulsed, as in standard proton synchrotrons, by having the magnetic field increase rapidly with radius. This was impossible in a weak focusing machine, because of the necessity of keeping $n$ between zero and one, but quite allowable if strong focusing is used.

The non-pulsed character of the magnetic field allows "beam stacking," the adding together of a number of separately injected 
and accelerated beams, which makes possible a very high current accelerator.

A number of electron models have been built to test FFAG, beam stacking, and different types of design. Fig. 14 shows a plan view of a radial-sector type electron model. The magnetic fields increase with radius, so in order to get alternating gradient focusing, alternate magnets must have negative magnetic fields-as indicated where the electrons are bending away from the center of the machine. Fig. 15 shows the electron energy and magnitude of the magnetic field as a function of the radius, and Fig. 16 is a picture of the model.

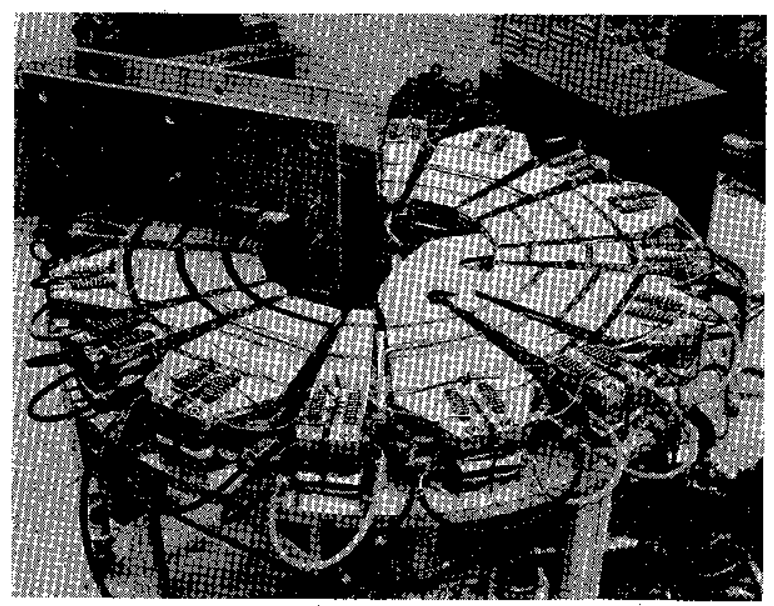

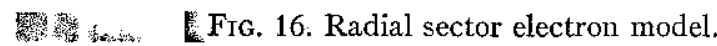

Because of the large scalloping of the orbits, it is possible to make a machine with positive and negative magnets the same-and accelerate particles in both directions simultaneously. A number of pulses could be "stacked" at full energy. The two oppositely directed beams of particles then could be made to collide-giving a considerably larger center-of-mass energy than possible with a collision with a stationary target. Fig. 17 shows such a two-way $50 \mathrm{Mev}$ electron model being built at MURA. The magnetic fields are being trimmed to the correct values and it should operate with a high beam current early in 1961. If the current is high enough, electron-electron collisions will be studied. Fig. 18 shows a plan view of a possible high energy two-way synchrotron. Because of the negative fields, the machine would be large. A $15 \mathrm{Bev}$ accelerator of this type would have a radius of about 600 feet, but the $30 \mathrm{Bev}$ center-of-mass energy would be the equivalent of that obtained by a conventional accelerator of $540 \mathrm{Bev}$.

Another type of FFAG accelerator is the spiral-ridge type, pro- 


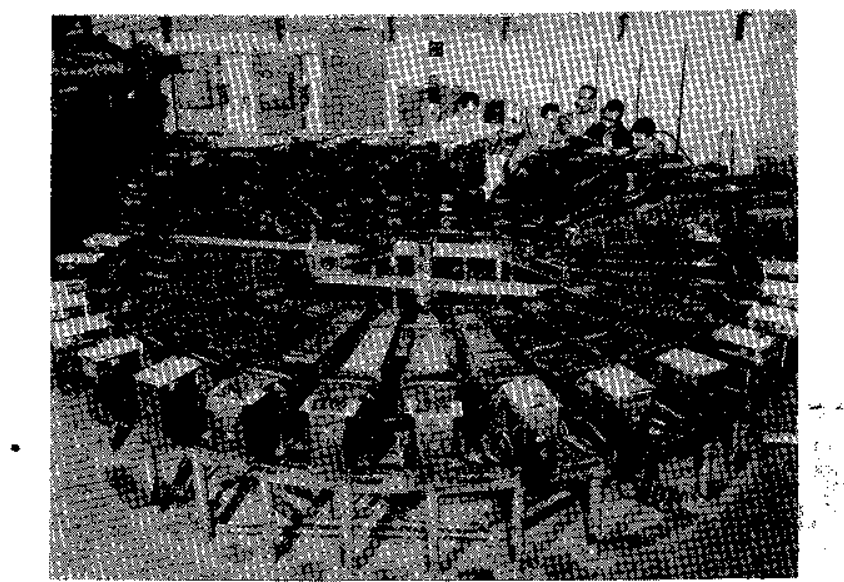

Fig. 17. $50 \mathrm{Mev}$ two-way electron model.

posed by Kerst. If the maxima of the fields follow spiral curves, a particle crossing the spiral will experience alternating gradient focusing. The fields can be made to increase with radius, without the necessity of negative fields - so the machine can be made considerably smaller than the radial sector type. Fig. 19 shows an electron model of the spiral ridge type. Of course, two-way acceleration is not possible here. A way of obtaining colliding beams using a one-way accelerator is with the use of two intersecting storage rings, as indicated in Fig. 20.

These new accelerators are still in the study and model stage. No large scale construction of such a multi-Bev machine is under way as yet.

\section{NATIONAL CENTER FOR RESEARCH ON ATMOSPHERE ESTABLISHED}

A new national laboratory to probe basic weather processes and other kinds of atmospheric behavior was formally established on Dec. 13 in Boulder, Colorado.

Known as the National Center for Atmospheric Research, or NCAR, the laboratory is run by a 14-university corporation under the sponsorship of the National Science Foundation. The Center was established as a result of a report to the National Academy of Sciences calling for a major national effort in the atmospheric sciences.

This report urged that the National Center tackle problems involving amounts of manpower and facilities beyond the capacity of individual universities to support.

The legal proceedings on Dec. 13 also marked the merger of the High Altitude Observatory, which has operated solar and astrophysical research facilities in Boulder and Climax for the past 15 years, with the university corporation. Dr. Walter Orr Roberts, director of the High Altitude Observatory since it was founded, was appointed director of the new National Center in June, 1960. 